\title{
Fuzzy Topology, Quantization and Gauge Fields
}

\section{Sergey Mayburov*}

Lebedev Institute of Physics

E-mail: mayburovesci.lebedev.ru

It was argued earlier that Dodson-Zeeman fuzzy topology $(\mathrm{FT})^{1}$ represents the possible mathematical basis for quantum space-time structure ${ }^{2}$. Here the quantization formalism related to it will be described ${ }^{3,4}$.

As the example, the quantization of massive particles is considered, it's shown that the coordinate uncertainty is generic in FT. FT fundamental set $D$ is Poset $^{3,4}$, so that some its element pairs in place of standard ordering relation $d_{j} \leq d_{k}$, can obey to incomparability relation: $d_{l} \sim d_{m}$. For illustration, consider discrete Poset $D=A^{p} \cup B$, which includes the subset of incomparable elements $A^{p}=\left\{a_{j}\right\}$, and the subset $B=\left\{b_{i}\right\}$ which is maximal totally ordered $D$ subset. $B$ indexes grow correspondingly to their ordering, i.e. $\forall i, b_{i} \leq b_{i+1}$. Suppose that for some $a_{j}$ and $B$ interval $\left\{b_{l}, b_{n}\right\}, a_{j} \sim b_{i} ; \forall i: l \leq i \leq n$. In this case $a_{j}$ is "smeared" over $\left\{b_{l}, b_{n}\right\}$ interval, which is analogue of $a_{j}$ coordinate uncertainty, if to regard $B$ as $D$ "coordinate axe". Analogously to it, 1-dimensional model Universe corresponds to Poset $U=A^{p} \cup X$ where $A^{p}$ is the massive particle subset, $X$-continuous ordered subset $R^{1}$, which describes 1-dimensional euclidian geometry. If for some $a_{j}$ and $X$ interval $\left\{x_{c}, x_{d}\right\}$ the relation $a_{j} \sim x_{b}$ holds for all $x_{b} \in\left\{x_{c}, x_{d}\right\}$, then $a_{j}$ possess $x$-coordinate uncertainty of the order $\left|x_{d}-x_{c}\right|$. To detalize $a_{j}$ characteristics, the corresponding fuzzy weight $w_{j}(x) \geq 0$ introduced with the norm $\left\|w_{j}\right\|=1$, so that $w_{j}(x)$ value indicates where on $X$ axe $a_{j}$ is mainly concentrated ${ }^{3}$. In this framework $a_{j}$ corresponds to the formal definition of fuzzy point and $U$ of fuzzy set ${ }^{3}$.

In such approach massive particle $m$ can be described as the evolving fuzzy point $a_{i}(t)$ of $U$. It's shown then that the corresponding normalized $m$ density $w(x, t)$ evolves according to the flow continuity equation: $\frac{\partial w}{\partial t}=-\frac{\partial(w v)}{\partial x}$ where $v(x, t)$ is $w$ flow local velocity. The independent $m$ parameters $w(x), v(x)$, which characterize $m$ state, can be unambiguously mapped to normalized complex function $\varphi(x)$. Assuming space-time shift invariance, it's proved that $\varphi(x, t)$ evolution obeys to Schrödinger equation for arbitrary $m$ mass $\mu$, such theory can be also extended for 3-dimensional case $^{4}$. It's proved also that in relativistic case $m$ evolution described by Dirac equation for spin $\frac{1}{2}$. Particle's interactions on such fuzzy manifold are shown to be gauge invariant, the interactions of fermion muliplets are performed by Yang-Mills fields ${ }^{5}$.

References

[1] Dodson, C.J.T. (1974), Bull. London Math. Soc. 6, 191

[2] Mayburov, S. (2008), J. Phys. A 41, 164071

[3] Bandmeyer, H. and Gottwald, S. (1995), Fuzzy Sets, Fuzzy Logics, Fuzzy Methods with Applications (Wiley, N-Y)

[4] Mayburov S. (2012) Phys. Part. Nucl. (2012) 43, 465 ; hep-th:1205.3019

[5] Mayburov S. (2010) Int. J. Theor. Phys. 49, 3192

Frontiers of Fundamental Physics 14 - FFP14,

15-18 July 2014

Aix Marseille University (AMU) Saint-Charles Campus, Marseille

* Speaker. 